Die Verfasserin hat eine emsig recherchierte und überzeugend argumentierende Untersuchung vorgelegt, die eine Vielzahl von Aspekten einbezieht - und damit Schwachstellen im Forschungsstand sichtbar macht. Entschied das von den Alliierten in Gang gesetzte Prüfverfahren ,,anhand der politischen Vergangenheit einer Person über deren gegenwärtiges Bedrohungspotenzial für die Nachkriegsgesellschaft" (S. 458), so reagierten die Betroffenen darauf mit Deutungsmustern, mit denen sie trotz ihrer ideologischen Übereinstimmung und organisatorischen Eingebundenheit innerlichen Abstand zum Nationalsozialismus beanspruchten. Solche Distanzierung ,und die Hinwendung zur Demokratie in den 1950er Jahren [vollzog sich] zugleich durch kritische und nicht-kritische Formen der Vergangenheitsthematisierung“ (S. 473). Bestimmend waren aber nicht aufrichtige Selbstkritik und Schuldeingeständnis, sondern ,eine Form biografischen Erzählens, mit der sich Distanz zum Nationalsozialismus glaubhaft behaupten und zugleich das eigene Leben trotz des Engagements für die NS-Diktatur als kontinuierlich begreifen ließ“ (S. 487f.).

Somit ist die politische Säuberung der frühen Jahre nach dem Nationalsozialismus keineswegs an Lügen und Täuschungsmanövern der Betroffenen gescheitert. Und von nun an werden dank Leßaus Studie diejenigen, die sich an die Auswertung von Entnazifizierungsakten machen, an ihre Arbeit mit anderen Augen herangehen.

\title{
Pohl, Natalie: Atomprotest am Oberrhein. Die Auseinandersetzung um den Bau von Atomkraftwerken in Baden und Elsass (1970-1985), (Schriftenreihe des Deutsch-Französischen Historikerkomitees, Bd. 15), 443 S., Franz Steiner Verlag, Stuttgart 2019.
}

\section{Stephen Milder}

Angenommen: 24. März 2021 / Online publiziert: 15. April 2021

(C) Der/die Autor(en) 2021

Schon 1980 hat der Soziologe Dieter Rucht den südbadischen Ort Wyhl am Kaiserstuhl mit seinem Buch Von Wyl nach Gorleben zum Ursprungspunkt der bundesdeutschen Anti-Atombewegung gekürt. In den letzten Jahren ist die Bedeutung der

S. Milder $(\triangle)$

Ludwig-Maximilians-Universität München, München, Deutschland

E-Mail: stephen.milder@rcc.lmu.de 
Auseinandersetzung um das geplante Kernkraftwerk Wyhl für die Weiterentwicklung der deutschen Anti-Atombewegung, die transnationale Vernetzung der Atomkraftgegner, und die Demokratisierung Deutschlands von mehreren Historiker_innen untersucht worden. Nun legt Natalie Pohl mit ihrer im Cotutelle-Verfahren an der Universität des Saarlandes und der Université Paris-Sorbonne geschriebenen Dissertation eine Studie vor, die Wyhl in seinen regionalen Kontext - sprich den „Atomprotest am Oberrhein“ - stellt, und diesen Themenkomplex ,als ganzes untersucht“" (S. 18). Da der Oberrhein die Grenze zwischen Südbaden und dem Elsass bildet, ist Pohls regionale Studie gleichzeitig ein internationaler Vergleich, der die „Charakteristika beziehungsweise Unterschiede und Gemeinsamkeiten der Anti-AtomkraftBewegung in beiden Ländern“ (Ebd.) herausarbeiten will.

Pohl untersucht den Atomprotest in Südbaden und Elsass systematisch und flächendeckend. Zwischen einer Einführung, die ihre Vorgehensweise erläutert, und einer kurzen, zusammenfassenden Schlussbetrachtung, ist das Buch in fünf stichhaltige Kapitel organisiert. Die ersten drei Kapitel, die die regionalen Bürgerinitiativen vorstellen und die Auseinandersetzungen um den Bau von Kernkraftwerken schildern, bilden die Basis der Studie. Im Gegensatz zu anderen kürzlich erschienenen Studien der Anti-Atombewegung, die den Transfer zwischen nationalen Kontexten hervorheben, konzentriert sich Pohl eher auf den Vergleich der Entwicklungen in den beiden Ländern. Pohl macht zum Beispiel die unterschiedliche Zusammensetzung der Atomkraftgegner auf den beiden Rheinseiten deutlich: während das elsässische Comité pour la Sauvegarde de Fessenheim et de la plaine du Rhin ,über klare Strukturen und Vereinsstatuten“ (S. 209) verfügte, hatten die meisten badische Bürgerinitiativen „gemeinhin einen niedrigen Organisationsgrad“ (S. 212). Auch die Rolle einzelner lokaler Gruppierungen aus Baden und Elsass innerhalb der regionalen Dachorganisation, der Badisch-Elsässischen Bürgerinitiative, wird auf vergleichende Weise dargestellt. Insofern kann Pohl konstatieren, dass die Badisch-Elsässische Bürgerinitiative in vielen Aspekten eher ein badischer als ein internationaler Verband war.

Auch die Hintergründe und der weitere Verlauf der Auseinandersetzungen werden anhand von Quellen aus 13 Archiven in Deutschland und Frankreich fast enzyklopädisch dargestellt. Die Perspektiven weniger, besonders ,,gesprächiger“ Atomkraftgegner - nämlich die des Liedermachers Walter Mossmann, des Pfarrers Günter Richter, des Friedensforschers Wolfgang Sternstein und des Deutschlehrers JeanJacques Rettig, die in Memoiren und in archivalischen Unterlagen (und im Fall Rettigs über ein Zeitzeugeninterview) reichlich übermittelt sind - sind besonders betont und bilden eine Art roten Faden durch das umfangreiche Kapitel. Dadurch werden bewegungsinterne Diskussionen oder auch die Abläufe bestimmter Ereignisse, wie zum Beispiel die initiale Besetzung des Reaktor-Bauplatzes in Wyhl, großteils nur einseitig dargestellt. Dagegen werden Auseinandersetzungen innerhalb der Regierung Baden-Württembergs, oder zwischen Lokalpolitikern und der Parteiprominenz der CDU, durch weitreichende archivalische Forschung und die Hervorhebung wiedersprechender Stimmen besonders glaubwürdig dargestellt.

Die beiden letzten Kapitel erweitern die Untersuchung der Bewegung durch die Verwendung von „kultur-, medien-, und erinnerungsgeschichtlicher Perspektive“ (S. 19). Besonders gelungen sind die Besprechungen der Berichterstattung in her- 
kömmlichen Medien und der Gegenöffentlichkeit der Bürgerinitiativen. Hier zeigt Pohl inwiefern die Berichterstattung der Badischen Zeitung die Anti-Atombewegung intensiver und freundlicher behandelte als die der Zeitung Dernières Nouvelles d'Alsace. Die Gegenöffentlichkeit der Atomgegner wird anhand von Studien der Volkshochschule Wyhler Wald und des Radio Verte Fessenheim untersucht. Pohl achtet besonders auf die Entstehung der Institutionen in der heißen Phase der AntiAtombewegung um 1975, aber auch auf die langfristige Weiterentwicklung. So stellt ihre Geschichte des Piratensenders Radio Verte Fessenheim, der sich über die Jahre zu dem lizensierten Sender Radio Dreyeckland entwickelte, einerseits die Möglichkeiten der nahen Grenze, und anderseits die Schwierigkeiten ein lokales und gleichzeitig internationales Radioprogramm zu erstellen, dar.

Pohls Diskussionen der kulturellen Seiten des Protests sind auch äußerst ergiebig. Sie zeigt, wie Protestlieder die Vielfalt der Bewegung widerspiegelten, und findet, dass „das gemeinsame Singen [...] ein Gemeinschaftsgefühl [erzeugte]“ und „die Regionale Dimension der Proteste“ (S. 392) unterstrich. Auch wiederentdeckte Widerstandsgeschichten aus der Region, wie der Bauernkrieg des 16. Jahrhunderts, die Salpeterer-Unruhen des 18. und 19. Jahrhunderts, und sogar die Revolution von 1848, würden von den Atomkraftgegnern verwendet, um eine gemeinsame - und obrigkeitskritische - regionale Identität zu erstellen.

Es ist vor allem die Fülle von Details, die lange Zeitspanne - die weit über die heiße Phase der Bewegung in der Mitte der 1970er Jahre hinaus geht - und der nuancierte Vergleich der Ereignisse im Elsass mit denen in Südbaden, die Pohls Studie auszeichnen. Insofern gelingt es ihr, die historische Darstellung des Atomprotests am Oberrhein maßgeblich zu bereichern. Mit einer deutlicheren, etwas tiefer in der Historiographie verwurzelten Argumentation wären ihre weitreichenden Befunde über das Geschehen im Elsass und in Baden für die aktuelle Debatte über die Entstehung, die Wirkung, und die langfristige Bedeutung der Anti-Atombewegung der 1970er Jahre insgesamt noch aussagekräftiger.

Funding Open Access funding enabled and organized by Projekt DEAL.

Open Access Dieser Artikel wird unter der Creative Commons Namensnennung 4.0 International Lizenz veröffentlicht, welche die Nutzung, Vervielfältigung, Bearbeitung, Verbreitung und Wiedergabe in jeglichem Medium und Format erlaubt, sofern Sie den/die ursprünglichen Autor(en) und die Quelle ordnungsgemäß nennen, einen Link zur Creative Commons Lizenz beifügen und angeben, ob Änderungen vorgenommen wurden.

Die in diesem Artikel enthaltenen Bilder und sonstiges Drittmaterial unterliegen ebenfalls der genannten Creative Commons Lizenz, sofern sich aus der Abbildungslegende nichts anderes ergibt. Sofern das betreffende Material nicht unter der genannten Creative Commons Lizenz steht und die betreffende Handlung nicht nach gesetzlichen Vorschriften erlaubt ist, ist für die oben aufgeführten Weiterverwendungen des Materials die Einwilligung des jeweiligen Rechteinhabers einzuholen.

Weitere Details zur Lizenz entnehmen Sie bitte der Lizenzinformation auf http://creativecommons.org/ licenses/by/4.0/deed.de. 